\title{
On Some Functionals of Laplacian Processes
}

\author{
R. Fortet
}

\begin{abstract}
Let $X(t)$ be a random function derived, in a sense that is explained in the paper, from a Poisson process. It is proved that, under certain assumptions, the distribution of the functional $\int_{t}^{t+T} V(u, X(u)) d u$ tends to a Laplacian (i. e. normal) distribution as $T \rightarrow \infty$. This result is extended to the case that $X(t)$ is itself a Laplacian process by means of a theorem stating that, under certain assumptions, every Laplacian process is the limit of some random function derived from a Poisson process.
\end{abstract}

We shall use the following abbreviations:

fr: function of repartition.

cf: characteristic function.

c: covariance.

fc: function of correlation.

$\mathrm{rf}$ : random function.

rv: random variable.

$\mathrm{mq}$ : in quadratic mean.

ac: almost certain, with probability 1 .

lp: Laplacian process.

rfP: random function derived from a Poisson process.

em: mathematical expectation.

emq: standard deviation.

imq: integral in quadratic mean.

iac: integral with probability 1.

Section 1 is devoted to the statement of some lemmas; section 2 is to show that, under some general assumptions, a lp may be considered as the limit, in law, of certain rfP's; section 3 is to state our principal result in the stationary case; section 4 is devoted to extensions of these results to nonstationary cases; in section 5, we indicate some generalizations in another direction.

1. Let $Y(t)$ be a real rf of second order defined over $(-\infty,+\infty)$, the c $\gamma(t, \tau)$ of which is $R$-integrable (on every finite domain); we assume $E[Y(t)] \equiv 0$; hence:

$$
L(t, \tau)=\int_{t}^{\tau} Y(u) d u \quad \operatorname{imq}
$$

exists, has a null em and an emq $\sigma(t, \tau)$ given by:

$$
\sigma^{2}(t, \tau)=\int_{t}^{\tau} \int_{t}^{\tau} \gamma(u, v) d u d v^{2}
$$

we assume that: (a) there exists a fixed number $l$ such that, for any integers $n, m$, and for any values $t_{1}, \ldots, t_{n}, \tau_{1}, \ldots, \tau_{m}$ with:

$$
t_{1}<t_{2}<\ldots<t_{n}<\tau_{1}<\ldots<\tau_{m} \quad \tau_{1}-t_{n} \geqq l
$$

the two (n-dimensional and $m$-dimensional) rv $\left\{Y\left(t_{1}\right), \ldots, Y\left(t_{n}\right)\right\}$ and $\left\{Y\left(\tau_{1}\right), \ldots, Y\left(\tau_{m}\right)\right\}$ are independent; (b) there are two fixed positive numbers

1 The preparation of this paper was sponsored (in part) by the Office of Naval Research.

${ }_{2}^{2}$ Loeve, Fonctions aleatoires du second ordre, p. 320, in P. Levy, Processu Stochastiques, Paris, 1948, Gauthiers-Villars edit. $a$ and $b$ (independent of $t$ and $\tau$ ) such that

$$
a(\tau-t) \leqq \sigma^{2}(t, \tau) \leqq b(\tau-t)
$$

at least for $(\tau-t)>0$ and sufficiently large; (c) there is a finite positive function $K(u)$ such that:

$$
E|L(t, \tau)|^{3} \leqq K(\tau-t)
$$

for every $(t, \tau)$, at least for $(\tau-t)>0$ and sufficiently large.

Then we have the following lemma:

Lemma 1: Under the above assumptions (a), (b), (c), for every fixed $t$, the fr of $[L(t, t+T)] /[\sigma(t, t+T)]$ tends toward Laplace's law [with $\mathrm{em}=0$ and $\mathrm{emq}=1$ ] when $T$ tends toward $+\infty$; and $L(t, t+T) / T$ tends toward 0 in probability. ${ }^{3}$

Proof: Of course, it is sufficient to consider the case $t=0$; let $L$ be any positive number, larger than $l$; we put:

$$
\begin{aligned}
t_{n} & =n(L+l) & t_{n}^{\prime} & =n(L+l)-\boldsymbol{l} ; \\
X_{n} & =\int_{t_{n-1}}^{t_{n}^{\prime}} Y(u) d u & Y_{n} & =\int_{t_{n}^{\prime}}^{t_{n}} Y(u) d u
\end{aligned}
$$

and, if $n_{T}$ is the largest integer such that: $n_{T}(L+\boldsymbol{l}) \leqq$ $T$,

$$
Z_{T}=\int_{t_{n_{T}}}^{T} Y(u) d u
$$

We have:

$$
\begin{array}{r}
\frac{L(0, T)}{\sigma(0, T)}=\frac{1}{\sigma(0, T)} \cdot \sum_{j=1}^{n_{T}} X ;+\frac{1}{\sigma(0, T)} \cdot \sum_{j=1}^{n_{T}} Y_{j}+\frac{Z_{T}}{\sigma(0, T)} \\
E\left[\frac{Z_{T}^{2}}{\sigma^{2}(0, T)}\right] \leqq \frac{1}{a T} E\left[Z_{T}^{2}\right] \leqq \frac{b}{a} \frac{T-t_{n_{T}}}{T} \leqq \frac{b}{a} \frac{L+\boldsymbol{l}}{T}
\end{array}
$$

It must be pointed out that the $Y_{j}$ 's are mutually independent; also the $X_{j}$ 's are mutually independent; hence we have:

$$
E\left[\left(\frac{1}{\sigma(0, T)} \sum_{j=1}^{n_{T}} Y_{j}\right)^{2}\right] \leqq \frac{b}{a} \frac{\boldsymbol{l} n_{T}}{T} \leqq \frac{b}{a} \frac{\boldsymbol{l}}{L+\boldsymbol{l}}
$$

\footnotetext{
${ }^{3}$ We do not know if this rather obvious lemma is or is not already known.
} 
$l /(L+l)$ being a quantity that may be arbitrarily small, if we choose $L$ sufficiently large. From the independence of the $X_{j}{ }^{\prime}$ s, and from (b) and (c) it follows that the fr of

$$
\frac{U}{\sqrt{E\left[U^{2}\right]}}
$$

where

$$
U=\sum_{j=1}^{n_{T}} X_{j}
$$

tends toward Laplace's law [with $\mathrm{em}=0$ and $\mathrm{emq}=$ 1], when $T$ tends toward $+\infty$; on the other hand, it follows from (2) and (3) that $E\left[U^{2}\right] / \sigma^{2}(O, T)$ may be as near 1 as we like, if we choose $L$ and $T$ sufficiently large. The lemma follows obviously from these remarks.

Remark: It must be pointed out that, if $Y(t)$ is strictly stationary, the assumption (c) may be suppressed, the $X_{j}$ 's having, in such a case, the same fr; on the other hand, in the general case, (c) may, of course, be replaced by some other, more or less general, assumption of the same kind.

We consider now a real rf $Y(t)$, defined over $(-\infty$, $+\infty)$, stationary of second order and the fe $\gamma(h)$ of which is continuous; we are always considering

$$
L(t, \tau)=\int_{t}^{\tau} Y(u) d u
$$

but in this stationary case, $\sigma^{2}(t, \tau)$ depends only on $(\tau-t)$; we have the:

Lemma 2: For the existence of two positive numbers $a$ and $b$ such that:

$$
a(\tau-t) \leqq \sigma^{2}(\tau-t) \leqq b(\tau-t) \quad[(\tau-t)>0]
$$

for any $t, \tau$, at least for $(\tau-t)$ sufficiently large, it is ufficient that:

(a)

$$
\begin{gathered}
\int_{-\infty}^{+\infty}|\gamma(h)| d h<+\infty \\
\int_{-\infty}^{+\infty} \gamma(h) d h \neq 0
\end{gathered}
$$

If $F(\omega)$ is the spectral function of $\gamma(h)$, we have:

$$
\sigma^{2}(\tau-t)=2 \int_{-\infty}^{+\infty} \frac{1-\cos \delta \omega}{\omega^{2}} d F(\omega)
$$

where $\delta=\tau-t$; for any $\epsilon>0$, we have:

$$
\lim _{\delta \rightarrow+\infty} \frac{2}{\delta} \int_{|\omega|>\epsilon} \frac{1-\cos \delta \omega}{\omega^{2}} d F(\omega)=0 .
$$

Let us assume that, in the interval $(-\epsilon,+\epsilon), F(\omega)$ has a derivative $f(\omega)$, and that $f(\omega)$ is continuous for $\omega=0$, with $f(0) \neq 0$; we have:

$$
\begin{aligned}
& \int_{-\epsilon}^{+\epsilon} \frac{1-\cos \delta \omega}{\omega^{2}} d F(\omega)=f(0) \int_{-\epsilon}^{+\epsilon} \frac{1-\cos \delta \omega}{\omega^{2}} d \omega+ \\
& \int_{-\epsilon}^{+\epsilon} \frac{1-\cos \delta \omega}{\omega^{2}}[f(\omega)-f(0)] d \omega
\end{aligned}
$$$$
977170-52-3
$$

when $\delta \rightarrow+\infty, f(0) \int_{-\epsilon}^{+\epsilon} \frac{1-\cos \delta \omega}{2 \omega^{2}} d \omega$ is equivalent to $S \delta$, where $S$ is some fixed number $\neq 0$; and $\left|\int_{-\epsilon}^{+\epsilon} \frac{1-\cos \delta \omega}{\omega^{2}}[f(\omega)-f(0)] d \omega\right|$ is bounded by $\eta \delta$, where $\eta$ is a $>0$ number, as small as we like if we choose $\epsilon$ sufficiently small; on the other hand, $F(\omega)$ has an everywhere continuous derivative $f(\omega)$ if $\int_{-\infty}^{+\infty}|\gamma(h)| d h<+\infty$; and, as $f(0)=\int_{-\infty}^{+\infty} \gamma(h) d h$,

the lemma follows. Besides, we see that, under the assumptions (a) (b), there exists a number $c$ such as:

$$
\sigma^{2}(T) \sim c T
$$

when $T \rightarrow+\infty$; (5) is more precise than (4).

2. In what follows, we have to consider: a Poisson process $N(t)$ of constant parameter $m$; if $\tau>t$, $N(\tau)-N(t)$ is the number of the jumps between $t$ and $\tau$, and

$$
\operatorname{Pr}[N(\tau)-N(t)=K]=e^{-m(\tau-t) \frac{[m(\tau-t)]^{K}}{K !}} ;
$$

the derived centered process $N^{*}(t)=N(t)-m t$; a real function $R(t, \tau)$ of the two variables $t, \tau$, defined over $-\infty<t, \tau<+\infty$, such that, for every fixed $t$, $R^{2}(t, \tau)$ is, as a function of $\tau, L$-measurable and $L$ summable; the process $X(t)$ defined by:

$$
X(t)=\lim _{\alpha \rightarrow-\infty, \beta \rightarrow+\infty}\left\{\operatorname{iac} \frac{1}{\sqrt{m}} \int_{\alpha}^{\beta} R(t, \tau) d N^{*}(\tau)\right\} .
$$

We shall call such a process $X(t)$ a random function derived from a Poisson process (rfP). The precise meaning of (6) is stated, for instance, by Fortet. ${ }^{4}$ It is known (see footnote 4) that:

Theorem I: If $m \rightarrow+\infty, X(t)$ is tending in law ${ }^{5}$ toward the Laplacian process $X^{*}(t)$, the covariance $\Gamma(t, \tau)$ of which is given by:

$$
\Gamma(t, \tau)=\int_{-\infty}^{+\infty} R(t, u) R(\tau, u) d u
$$

Reciprocally, one may ask under what conditions a real Laplacian process $X^{*}(t)$ with $c . \Gamma(t, \tau)$ may be considered as the limit in law of some $\mathrm{rfP}$. We gave (see footnote 4) a partial answer to this question, by the following:

Theorem II: Let the c $\Gamma(t, \tau)$ of $X^{*}(t)$ be a function $r(h)$ of $h=\tau-t$ only; $X^{*}(t)$ is the limit in law of a rfP with a $R(t, \tau)=R(\tau-t)$ depending on $(\tau-t)$ only, if and only if: (a) $r(h)$ [which is necessarily positive definite as a c] is continuous [hence, it is a ef] with an absolutely continuous spectral function; (b) $R(u)$ is of the form:

${ }_{4}^{4}$ Fortet, Random functions from a Poisson process, Berkeley Second Symposium on Mathematical Statistics and Probability (1950).

5 A process $X(t)$ tends toward the process $X^{*}(t)$ in law if, for any $n$ and any $t_{1}$ $t_{2}, \ldots \ldots, t_{n}$, the fr of the ( $n$-dimensional) $\mathrm{rv}\left[\boldsymbol{X}\left(t_{1}\right), X\left(t_{2}\right), \ldots \ldots, \boldsymbol{X}\left(t_{n}\right)\right]$ is tending toward the fr of $\left[X^{*}\left(t_{1}\right), X^{*}\left(t_{2}\right), \ldots, X^{*}\left(t_{n}\right)\right]$. 
$R(u)=$

$$
\frac{1}{\sqrt{2 \pi}} \int_{-\infty}^{+\infty} \sqrt{f(\omega)} e^{i \psi(\omega)} e^{-i \omega u} d \omega \text { [Fourier-Plancherel }
$$

where $f(\omega)$ is the spectral density of $r(h)$, and $\psi(\omega)$ is any odd function.

We shall give here a more detailed proof of this theorem; if there is a $\operatorname{rfP} X(t)$ with $R(t, \tau)=R(\tau-t)$ depending on $(\tau-t)$ only, $[$ and such that we have: $\left.\int_{-\infty}^{+\infty} R^{2}(t, \tau) d \tau=\int_{-\infty}^{+\infty} R^{2}(u) d u<+\infty\right]$ and tending in law toward $X^{*}(t)$, we must have by $(7)$ :

$$
r(h)=\int_{-\infty}^{+\infty} R(u) R(u+h) d u
$$

Let us put:

$a(\omega) e^{i \psi(\omega)}=\frac{1}{\sqrt{2 \pi}} \int_{-\infty}^{+\infty} R(u) e^{-i \omega u} d u \begin{gathered}{[\text { Fourier-Plancherel }} \\ \text { transform] }\end{gathered}$

$a(\omega)$ being real, even, and $\geqq 0 ; \psi(\omega)$ is odd, $R(u)$ being real; then we have (see ${ }^{6}$ for instance):

$$
\begin{aligned}
r(h) & =\int_{-\infty}^{+\infty} a(\omega) e^{i \psi(\omega)} a(-\omega) e^{i \psi(-\omega)} e^{-i h \omega} d \omega \\
& =\int_{-\infty}^{+\infty} a^{2}(\omega) e^{-i h \omega} d \omega .
\end{aligned}
$$

Hence, $r(h)$ is necessarily continuous and with a spectral density $a^{2}(\omega)$. Conversely, if $r(h)$ is continuous and has a spectral density $f(\omega)$, a function $R(u)$ exists given by:

$$
R(u)=\int_{-\infty}^{+\infty} \frac{1}{\sqrt{2 \pi}} \sqrt{ } f(\omega) e^{1 \psi(\omega)} e^{-i \omega u} d \omega
$$

where $\psi(\omega)$ is any odd function, such that:

$$
\int_{-\infty}^{+\infty} R^{2}(u) d u<+\infty
$$

and satisfying (8).

It is more difficult to obtain a result in the general case; of course, $\Gamma(t, \tau)<+\infty$ for every $t$ is a necessary condition; we suppose it satisfied in what follows; in a heuristic manner, we may develop the following considerations: $\Gamma(t, \tau)$ is, as a $c$, of the nonnegative type (see reference cited in footnote 2, p. 301); hence, if $H$ is the linear operator defined on $L^{2}(-\infty,+\infty)$ by

$$
g(t)=H[f]=\int_{-\infty}^{+\infty} \Gamma(t, \tau) f(\tau) d \tau
$$

then, under some general assumptions, $H$ is selfadjoint positive (bounded or not); hence it has ${ }^{7}$ a self-adjoint positive square root $K$ [and only one ${ }^{8}$ ],

${ }^{6}$ E. C. Titchmarsh, Introduction to the theory of Fourier integrals, 2d ed. Clarendon Press, Oxford, 1948).

7 Bela de Nagy, Spektral darstellung lineares Transformationen des Hilbertschen Raumes, Ergebnisse der Math. 5, part 5, p. 52 (Springer, Berlin, 1942).

${ }^{8} \mathrm{But}$ in general there are also self-adjoint nonpositive square roots, and also non self-adjoint square roots. that is to say that: $K^{2}=H$; under some general assumptions, $H$ being an integral operator, $K$ is also an integral operator and admits a representation of the form:

$$
g(t)=K[f]=\int_{-\infty}^{+\infty} K(t, \tau) f(\tau) d \tau
$$

The relation $K^{2}=H$ is expressed by:

$$
\Gamma(t, \tau)=\int_{-\infty}^{+\infty} K(t, u) K(u, \tau) d u
$$

and, because $K(t, \tau)$ is necessarily symmetric, by:

$$
\Gamma(t, \tau)=\int_{-\infty}^{+\infty} K(t, u) K(\tau, u) d u
$$

Hence we have found a solution of (7) (and a symmetric one): we may take $R(t, \tau)=K(t, \tau)$; and there is at least one $\mathrm{rfP}$, the limit in law of which is $X^{*}(t)$. For instance, if $\Gamma(t, \tau)$ is continuous over every finite domain and if

$$
\int_{-\infty}^{+\infty} \int_{-\infty}^{+\infty} \Gamma^{2}(t, \tau) d t d \tau<+\infty
$$

$H$ is completely continuous; all the necessary assumptions are satisfied; $\Gamma(t, \tau)$ admits the representation:

$$
\Gamma(t, \tau)=\sum_{j} \frac{f_{j}(t) f_{j}(\tau)}{\lambda_{j}}
$$

where the $\lambda_{j}$ 's are the eigenvalues of $H$, and $f_{j}$ 's its normed eigenfunctions; we have:

$$
R(t, \tau)=K(t, \tau)=\sum_{j} \frac{f_{j}(t) f_{j}(\tau)}{\sqrt{\lambda_{j}}} \text { in } \mathrm{mq}
$$

Then we may remark that, if we have a solution $R^{0}(t, \tau)$ of (7) for a particular $\Gamma(t, \tau)=\Gamma^{0}(t, \tau), R(t, \tau)=$ $f(t) R^{0}(t, \tau)$ is a solution of $(7)$ for $\Gamma(t, \tau)=f(t) f(\tau) \Gamma^{0}(t, \tau)$. From that we may deduce that for every $\Gamma(t, \tau)$ continuous on every bounded domain, (7) admits at least one solution (symmetric or not); the reason is that, in such a case, we may find a continuous function $f(t)$ and a kernel $\Gamma^{0}(t, \tau)$ such that: (a) $\Gamma^{0}(t, \tau)$ is continuous (on every bounded domain); (b) $\int_{-\infty}^{+\infty}$ $\int_{-\infty}^{+\infty}\left[\Gamma^{0}(t, \tau)\right]^{2} d t d \tau<+\infty ; \quad$ (c) $\Gamma(t, \tau)=f(t) f(\tau) \Gamma^{0}(t, \tau)$. For instance, we may choose $f(t)$ in the following way: let $\lambda(\alpha)$ be the $\nmid u b|\Gamma(t, \tau)|$ when $0 \leqq|\tau| \leqq|t| \leqq$ $\alpha, \lambda^{\prime}(\alpha)=\max [1, \lambda(\alpha)]$; we take: $f(t)=\lambda^{\prime}(t) e^{t^{2}}$. Hence we have the:

Theorem III: Every Laplacian process $X^{*}(t)$, the c $\Gamma(t, \tau)$ of which is continuous on every bounded domain is the limit in law (as $m$ is tending toward $+\infty$ ) of at least one rfP.

On the other hand, it is obvious that in general (7) admits several, and even an infinity, of solutions 
$R(t, \tau)$ : for instance, if $\Gamma(t, \tau)=1$, there is an infinity of choices for $R(t, \tau)$ which are functions of $\tau$ only that satisfy (7); another remark is that a $\Gamma(t, \tau)$, given by (7) when $R(t, \tau)$ is given, is necessarily a covariance, but is not necessarily a continuous one [see, for example, the case where:

$$
\left.R(t, \tau)=0 \text { if } t=0 \text { and: }=e^{-\tau} \text { if } t \neq 0\right]
$$

3. In what follows, we consider $\operatorname{rfP} X(t)$ like (6); and, $V(t, x)$ being any real, given function of $t$ and $x$, we consider the rf

$$
Y(t)=V[t, X(t)]
$$

and the functionals:

$$
L(t, \tau)=\int_{t}^{\tau} V[u, X(u)] d u
$$

and our aim is to prove that, under some assumptions, the fr of $L(t, t+T)$, when this $\mathrm{rv}$ is properly normed, and for any fixed $t$, tends toward Laplace's $\mathrm{fr}$. when $T \rightarrow+\infty$. And we shall try to extend this result to the case where $X(t)$ is not a rfP but a Laplacian process. In all cases we shall assume that there is a finite positive number $M$, independent of $t$ and $x$ (but, of course, depending on the considered function $V$ ) such that for any $t, x, x^{\prime}$ :

$$
\left|V(t, x)-V\left(t, x^{\prime}\right)\right| \leqq M\left|x-x^{\prime}\right|^{\alpha}
$$

where $\alpha$ is any fixed number with $0<\alpha \leqq 1$. Of such a function $V$, we shall say that it belongs to the class $C_{\alpha}$; if $V \epsilon C_{\alpha}$ and if $f(t)$ is any function of $t$, it is obvious that $V+f$ belongs to $\dot{C}_{\alpha}$.

In this section, we restrict ourselves to the stationary case, in which $R(t, \tau)=R(\tau-t)$ depends on $(\tau-t)$ only, and $V(t, x)=V(x)$ depends on $x$ only; we begin by considering the case where $X(t)$ is a rfP.

It is readily seen that $Y(t)$ is strictly stationary, that $E[Y(t)], \quad E[|Y(t)|], E\left[Y^{2}(t)\right], \quad E[Y(t) Y(t+h)]=$ $\gamma(h)$ exist; from a preceding remark, we may assume, without loss of generality, that $E[Y(t)]=0 ; \gamma(h)$ is the fc of $Y(t)$, and it is easy to prove that $\gamma(h)$ is continuous; $Y(t)$ is continuous in $\mathrm{mq}$ and

$$
L(t, \tau)=\int_{t}^{\tau} V[X(u)] d u \quad \operatorname{imq}
$$

exists; $E[L(t, \tau)]=0$; the emq $\sigma(\tau-t)$ of $L(t, \tau)$ exists, depends only on $(\tau-\hat{t})$, and is given by:

$$
\sigma^{2}(\tau-t)=\int_{t}^{\tau} \int_{t}^{\tau} \gamma(u-v) d u d v
$$

It is supposed that:

$$
\int_{-\infty}^{+\infty} R^{2}(u) d u<+\infty
$$

We put:

$$
\phi(a)=\int_{|u|>a} R^{2}(u) d u
$$

and we assume that:

$$
\int_{-\infty}^{+\infty} \phi(a)^{\frac{\alpha}{2}} d a<+\infty
$$

We put:

$$
\begin{aligned}
& R_{a}(u)=\left\{\begin{array}{ll}
R(u) \text { if }|u| \leqq a \\
0 \quad \text { if }|u|>a
\end{array} \quad R_{a}^{\prime}(u)=R(u)-R_{a}(u)\right. \\
& X_{a}(t)=\lim _{\alpha \rightarrow-\infty, \beta \rightarrow+\infty}\left\{\operatorname{iac} \frac{1}{\sqrt{m}} \int_{\alpha}^{\beta} R_{a}(t-\tau) d N^{*}(\tau)\right\} \\
& X_{a}^{\prime}(t)=X(t)-X_{a}(t)= \\
& \lim m g_{\alpha \rightarrow-\infty, \beta \rightarrow+\infty}\left\{\operatorname{iac} \frac{1}{\sqrt{m}} \int_{\alpha}^{\beta} R_{a}^{\prime}(t-\tau) d N^{*}(\tau)\right.
\end{aligned}
$$

and we remark that: (a) if $|\tau-t| \geqq 2 a, X_{a}(t)$ and $X_{a}(\tau)$ are two independent rv; (b) for every fixed $t, X_{a}(t)$ and $X_{a}^{\prime}(t)$ are independent rv; but the two processes $X_{a}(t)$ and $X_{a}^{\prime}(t)$ are, in general, correlated, because $X_{a}(t)$ and $X_{a}^{\prime}(\tau)$ are not necessarily independent if $\tau \neq t$. Now we state the following lemma: let $R^{j}(u)(j=1,2, \ldots, r)$ be $r$ real functions such that:

$$
\int_{-\infty}^{+\infty}\left[R^{j}(u)\right]^{2} d u<+\infty \quad(j=1,2, \ldots, r) .
$$

We put:

$$
\phi_{j}(a)=\int_{|u|>a}\left[R^{j}(u)\right]^{2} d u \quad(j=1,2, \ldots, r)
$$

$$
\begin{aligned}
X^{j}(t)= & \operatorname{limmq}_{\alpha \rightarrow-\infty, \beta \rightarrow+\infty} \\
& \left\{\operatorname{iac} \frac{1}{\sqrt{m}} \int_{\alpha}^{\beta} R^{j}(t-\tau) d N^{*}(\tau)\right\}(j=1,2, \ldots, r)
\end{aligned}
$$

Let $V_{j}(x)$ be $r$ functions of the class $C_{\alpha}$, such that:

$$
E\left\{V_{j}\left[X^{j}(t)\right]\right\}=0 \quad(j=1,2, \ldots, r)
$$

We put:

$$
Z(t)=\sum_{j=1}^{r} V_{j}\left[X^{j}(t)\right]
$$

Of course, $Z(t)$ is a strictly stationary $\mathrm{rf}$; let $\rho(h)=E[Z(t) Z(t+h)]$ be its fc. In what follows, $S$ is some finite fixed positive number, not necessarily the same in all the formulas; we have the following:

Lemma 3: $|\rho(h)|=S\left(\sum_{j=1}^{r} \phi_{j}\left(\frac{h}{2}\right)^{\frac{\alpha}{2}}\right)$.

It is sufficient to prove this in the case $r=2 ; R_{a}^{j}(u)$, $R_{a}^{\prime \prime}(u), X_{a}^{i}(t), X_{a}^{\prime j}(t)$ being defined as in (13), (14), (14)', 
we put:

$$
Z_{a}(t)=V_{1}\left[X_{a}^{1}(t)\right]+V_{2}\left[X_{a}^{2}(t)\right]
$$

hen

$$
\begin{gathered}
E\left[Z_{a}(t)\right]=E\left[Z_{a}(t)-Z(t)\right] \\
\left.=E\left\{\left(V_{1} \mid X_{a}^{1}(t)\right]-V_{1}\left[X^{1}(t)\right]\right)+\left(V_{2}\left[X_{a}^{2}(t)\right]-V_{2}\left[X^{2}(t)\right]\right)\right\}
\end{gathered}
$$

and by (11):

$$
\left|E\left[Z_{a}(t)\right]\right| \leqq S\left\{E\left[\left|X_{a}^{\prime \prime}(t)\right|^{\alpha}\right]+E\left[\left|X_{a}^{\prime 2}(t)\right|^{\alpha}\right]\right\}
$$

By Hölder's inequality, we obtain:

because:

$$
\left|E\left[Z_{a}(t)\right]\right| \leqq S\left\{\phi_{1}(a)^{\frac{\alpha}{2}}+\phi_{2}(a)^{\frac{\alpha}{2}}\right\}
$$

$$
E\left\{\left[X_{a}^{\prime j}(t)\right]^{2}\right\}=\int_{-\infty}^{+\infty}\left[R_{a}^{\prime j}(u)\right]^{2} d u=\int_{|u|>a}\left[R^{j}(u)\right]^{2} d u=\phi_{j}(a)
$$

We put:

We have:

$$
\begin{aligned}
& A_{a}(t)=Z_{a}(t)-E\left[Z_{a}(t)\right] \\
& B_{a}(t)=E\left[Z_{a}(t)\right]+Z(t)-Z_{a}(t)
\end{aligned}
$$

$$
\begin{array}{r}
Z(t)=A_{a}(t)+B_{a}(t) \\
E\left[A_{a}(t)\right]=E\left[B_{a}(t)\right]=0
\end{array}
$$

and, if $|t| \geqq 2 a, A_{a}(0)$ and $A_{a}(t)$ are two independent r.v.; we have from (11) and (15):

$$
\left|B_{a}(t)\right| \leqq\left. S\left|\phi_{1}(a)^{\frac{\alpha}{2}}+\phi_{2}(a)^{\frac{\alpha}{2}}+\right| X_{a}^{\prime 1}(t)\right|^{\alpha}+\left|X_{a}^{\prime 2}(t)\right|^{\alpha} \mid
$$

Hence, from Hölder's inequalities:

$$
E\left(\left|B_{a}(t)\right|^{2}\right)=S\left[\phi_{1}(a)^{\frac{\alpha}{2}}+\phi_{2}(a)^{\frac{\alpha}{2}}\right]^{2}
$$

On the other hand, it is easy to see that:

$$
E\left(\left|A_{a}(t)\right|^{2}\right) \leqq S
$$

hence, if $a=h / 2$, we may write:

$$
\begin{aligned}
\rho(h)= & E[Z(0) Z(h)]= \\
& E\left[A_{\frac{h}{2}}(0) B_{\frac{h}{2}}(h)+A_{\frac{h}{2}}(h) B_{\frac{h}{2}}(0)+B_{\frac{h}{2}}(0) B_{\frac{h}{2}}(h)\right]
\end{aligned}
$$

(16), (17), (18) and Schwarz's inequality prove the Lemma 3. By exactly the same method, we may prove that:

\section{Lemma 4: If we put:}

$Y_{1}(t)=V_{1}\left[X^{1}(t)\right], \quad Y_{2}(t)=V_{2}\left[X^{1}(t)\right], \ldots, Y_{r}=V_{r}\left[X^{2}(t)\right]$ (under the same assumptions as above on the $X^{j}$ 's and the $V_{j}^{\prime}$ 's) and:

$$
E\left[Y_{j}(t) Y_{k}(t+h)\right]=\gamma_{j k}(h),
$$

we have:

$$
\left|\gamma_{j k}(h)\right| \leqq S\left[\sum_{j=1}^{r} \phi_{j}\left(\frac{h}{2}\right)^{\frac{\alpha}{2}}\right]
$$

(where $S$ may be chosen independent of $j$ and $k$ ).

Now, we return to the rf $Y(t)$ defined by

$$
Y(t)=V[X(t)]
$$

and with fe $\gamma(h)$,

$$
L(t, \tau)=\int_{t}^{\tau} Y(u) d u
$$

By (9) and the lemma 3 (with $r=1$ ), we have:

$$
\int_{-\infty}^{+\infty}|\gamma(h)| d h<+\infty
$$

We shall assume that:

$$
\int_{-\infty}^{+\infty} \gamma(h) d h \neq 0
$$

It follows by the lemma 2 that there are two positive numbers $l$ and $m$ such as:

$$
l(\tau-t) \leqq \sigma^{2}(\tau-t) \leqq m(\tau-t) \quad(\tau>t)
$$

at least for $(\tau-t)$ sufficiently large; let $a$ be any positive number, we put:

$$
\begin{array}{rlrl}
Y_{a}(t) & =V\left[X_{a}(t)\right] & & \\
A_{a}(t) & =Y_{a}(t)-E\left[Y_{a}(t)\right] & B_{a}(t) & =Y(t)-Y_{a}(t)+ \\
& E\left[Y_{a}(t)\right] \\
L_{a}(t, \tau) & =\int_{t}^{\tau} A_{a}(u) d u & L_{a}^{\prime}(t, \tau)= & \int_{t}^{\tau} B_{a}(u) d u
\end{array}
$$

We have

$$
\begin{gathered}
Y(t)=A_{a}(t)+B_{a}(t) \\
L(t, \tau)=L_{a}(t, \tau)+L_{a}^{\prime}(t, \tau)
\end{gathered}
$$

and we remark that:

(a) if $|\tau-t| \geqq 2 a, A_{a}(t)$ and $A_{a}(\tau)$ are two independent rv; by lemma 3 , applied to $A_{a}(t)$, the fc $\gamma_{a}(h)$ of $A_{a}(t)$ is such as:

$$
\left|\gamma_{a}(h)\right| \leqq S \phi\left(\frac{h}{2}\right)^{\frac{\alpha}{2}}
$$

on the other hand we have:

$$
\lim _{a \rightarrow+\infty} \gamma_{a}(h)=\gamma(h)
$$

This follows obviously from:

$$
A_{a}(t)=Y(t)-\left[Y(t)-Y_{a}(t)\right]-E\left[Y_{a}(t)\right],
$$


from (12) applied to $Y_{a}(t)$, and from:

$$
\left|Y(t)-Y_{a}(t)\right|=\left|V[X(t)]-V\left[X_{a}(t)\right]\right| \leqq M\left|X_{a}^{\prime}(t)\right|^{\alpha} .
$$

It follows that, for any $a$ :

$$
\int_{-\infty}^{+\infty}\left|\gamma_{a}(h)\right| d h<+\infty
$$

and that, for any sufficiently large $a$, by (19):

$$
\int_{-\infty}^{+\infty} \gamma_{a}(h) d h \neq 0
$$

Hence for any sufficiently large $a$, by Lemma 2 , there are two positive numbers $l_{1}$ and $m_{1}$ such as:

$$
l_{1}(\tau-t) \leqq E\left[L_{a}(t, \tau)^{2}\right] \leqq m_{1}(\tau-t)
$$

and $A_{a}(t)$ being a strictly stationary process, it follows from Lemma 1 that, for any fixed $t$ and when $T$ is tending toward $+\infty$, the frof

$$
\frac{L_{a}(t, t+T)}{\sqrt{E\left[L_{a}(t, t+T)^{2}\right]}}
$$

tends toward Laplace's fr [with $\mathrm{em}=0$ and $\mathrm{emq}=1$ ]; on the other hand, putting:

$$
\alpha=u-v \quad \beta=u+v,
$$

we may write:

$\int_{t}^{t+T} \int_{t}^{t+T} \gamma(u-v) d u d v=\frac{1}{2} \int_{t \sqrt{2}}^{(t+T) \sqrt{2}}\left[\int_{\beta_{1}}^{\beta_{2}} \gamma(\alpha) d \alpha\right] d \beta$

where $\beta_{1}$ and $\beta_{2}$ are two functions of $\beta$, easy to determine; in the same way:

$\int_{t}^{t+T} \int_{t}^{t+T} \gamma_{a}(u-v) d u d v=\frac{1}{2} \int_{t \sqrt{2}}^{(t+T) \sqrt{2}}\left[\int_{\beta_{1}}^{\beta_{2}} \gamma_{a}(\alpha) d \alpha\right] d \beta$

From (20) and (21), it follows that:

$$
\lim _{a \rightarrow+\infty} \frac{\frac{1}{T} E\left(L_{a}(t, t+T)^{2}\right]}{\frac{1}{T} \sigma^{2}(T)}=1^{9}
$$

uniformly in $\mathrm{T}$.

(b) We may apply the lemma 3 to $B_{a}(t)$, in the following way: $r=2, \quad V_{1}[x]=V(x), \quad V_{2}(x)=V(x)-$ $E\left[Y_{a}(t)\right], R^{1}=R(u), R^{2}=R_{a}(u)$; as:

$$
\phi_{1}(u) \leqq \phi(u) \quad \phi_{2}(u) \leqq \phi(u),
$$

we obtain that, $\gamma_{a}^{\prime}(h)$ being the fe of $B_{a}(t)$ :

$$
\left|\gamma_{a}^{\prime}(h)\right| \leqq S \cdot \phi\left(\frac{h}{2}\right)^{\frac{\alpha}{2}} .
$$

\footnotetext{
${ }^{\ominus} \mathrm{It}$ is useful, for the proof, to divide the numerator and the denominator by $T$.
}

And, because:

$$
B_{a}(t)=\left\{V[X(t)]-V\left[X_{a}(t)\right]\right\}+E\left[Y_{a}(t)\right]
$$

it follows from (12) [applied to $Y_{a}(t)$ ] and from (8) that, for every fixed $h$ :

$$
\lim _{a \rightarrow+\infty} \gamma_{a}^{\prime}(h)=0
$$

From this we may deduce, as in (a), that:

$$
\lim _{a \rightarrow+\infty} \frac{1}{T} E\left[L_{a}^{\prime}(t, t+T)^{2}\right]=0
$$

uniformly in $\mathrm{T}$.

Now, from all these facts and from Lemma 1, it follows the:

Theorem IV: Under the above hypothesis, when $T$ tends toward $+\infty$ and for every fixed $t$, the fr of

$$
\frac{L(t, t+T)}{\sigma(T)}
$$

tends toward Laplace's fr [with $\mathrm{em}=0$ and $\mathrm{emq}=1$ ].

More generally, considering $Z(t)$ defined by:

$$
Z(t)=\sum_{j=1}^{r} V_{j}\left[X^{j}(t)\right]
$$

as above, under the same assumptions, and if we put:

$$
M(t, \tau)=\int_{t}^{\tau} Z(u) d u,
$$

obviously we obtain in the same way that:

Theorem $V$ : When $T$ tends toward $+\infty$, then, for every fixed $t$, the fr of

$$
\frac{1}{\sqrt{E\left[M(t, t+T)^{2}\right]}} M(t, t+T)
$$

tends toward Laplace's fr, at least if, $\delta(h)$ being the fc of $Z(t)$, we have:

$$
\int_{-\infty}^{+\infty} \delta(h) d h \neq 0
$$

On the other hand, considering $Y^{j}(t)=V_{j}\left[X^{j}(t)\right]$ $(j=1,2, \ldots, r)$, under the above assumptions, and assuming that, if $\gamma_{j}(h)$ is the fc of $Y_{j}(t)$, we have:

$$
\int_{-\infty}^{+\infty} \gamma_{j}(h) d h \neq 0 \quad(j=1,2, \ldots, r),
$$

putting:

$$
L^{j}(t, \tau)=\int_{t}^{\tau} Y^{j}(u) d u, \quad \sigma_{j}^{2}(\tau-t)=E\left[L^{j}(t, \tau)^{2}\right]
$$

it is easy to prove that:

Theorem VI: For every fixed $t$, as $T$ tends toward 
$+\infty$, the fr of the $r$-dimension $r v\left\{\frac{L^{1}(t, t+T)}{\sigma_{1}(T)}, \ldots\right.$, $\left.\frac{L^{r}(t, t+T)}{\sigma_{r}(T)}\right\}$ tends toward an $r$-dimensional Laplace's fr.

In order to prove theorem VI, we have to use Lemma 4, a reasoning very similar to that which gives Lemma 1, and to prove that, when $T \rightarrow+\infty$,

$$
E\left\{\frac{L^{1}(t, t+T)}{\sigma_{1}(T)} \times \frac{L^{2}(t, t+T)}{\sigma_{2}(T)}\right\}
$$

has a limit. That is not difficult, with (5) and a reasoning similar to that which leads to $(22){ }^{10}$

Extension to the case of a stationary Laplacian process: Let $X^{*}(t)$ be a Laplacian stationary process, with fe $r(h)$; from theorem II we know that $X^{*}(t)$ is the limit in law (when $m \rightarrow+\infty$ ) of the fdP $X(t)$ with $R(u)$ given by $(9)$; let us assume that there is a choice of $\psi(\omega)$ in $(9)$ in such a way that, $\phi(a)$ always being

we have:

$$
\int_{|u|>a} R^{2}(u) d u
$$

$$
\int_{-\infty}^{+\infty} \phi(a)^{\frac{\alpha}{2}} d a<+\infty
$$

Introducing, as above, $X_{a}(t)$ and $X_{a}^{\prime}(t)$, we know, by theorem I, or, better, by a more general theorem of [3] that the two-dimensional rf $\left\{X_{a}(t), X_{a}^{\prime}(t)\right\}$ tends in law toward a two-dimensional Laplacian rf $\left\{X_{a}^{*}(t)\right.$, $X_{a}^{\prime *}(t)$ ] (when $m \rightarrow+\infty$ ), and we see immediately that we may consider that:

$$
X^{*}(t)=X_{a}^{*}(t)+X_{a}^{\prime *}(t)
$$

It is clear that, if $|\tau-t| \geqq 2 a, X_{a}^{*}(t)$ and $X_{a}^{*}(\tau)$ are independent; that, for every $t, X_{a}^{*}(t)$ and $X_{a}^{\prime *}(t)$ are independent; but the two rf $\left.X_{a}^{*} t\right)$ and $X_{a}^{\prime *}(t)$ are correlated; the fc's of $X_{a}^{*}(t), X_{a}^{\prime *}(t)$, the correlations between $X^{*}(t)$ and $X_{a}^{*}(t), X^{*}\left(t\right.$ and $X_{a}^{*}(t), X_{a}^{*}(t)$ and $X_{a}^{\prime *}(t)$ being the same as those between $X(t)$ and $X_{a}^{a}(t), X(t)$ and $X_{a}^{\prime}(t), X_{a}(t)$ and $X_{a}^{\prime}(t)$. Whatever $m$ is, it is clear also that the preceding method may be applied to $X^{*}(t)$ as well as to $X(t)$, and without any change; in particular, we may conclude:

(a) from Lemma 3, that (24) is a representation if $X(t)$ be a sum of two Laplacian processes, the second of which is in some sense negligible if $a$ is large, the first being of a well-defined and very simple and special form; this decomposition is valid for a wide class of stationary Laplacian processes [it would be interesting to replace (23), which defines this class, by a more direct assumption on $r(h)$ ], and seems to us to be the most interesting feature which we encounter in this section; it must be pointed out that this decomposition is not a classical spectral decomposition; in such a spectral decomposition, which is valid for any stationary r.f. of second order, the

10 Theorem IV was first stated by Blanc-Lapierre, in Sur certaines fonctions aleatoires stationnaires, Thesis (Paris, 1945), Masson edit., but under some very aleatoires stationnaires, Thes
much stronger assumptions. terms of the sum are uncorrelated: but in (24), $X_{a}^{*}(t)$ and $X_{a}^{\prime *}(t)$ are correlated.

(b) Theorem IV and Theorem V are immediately applicable with $X^{*}(t)$ instead of $X(t)$; it is also possible to state the equivalent to Theorem VI.

4. Now, we take the case in which $R(t, \pi)$ is any function of $(t, \tau)$, but we assume that there is a nonnegative function $P(u)$ such as:

$$
|R(t, \tau)| \leqq R(t-\pi) \quad \int_{-\infty}^{+\infty} R^{2}(u) d u<+\infty
$$

and such that, if

$$
\begin{gathered}
\phi(a)=\int_{|u|>a} R^{2}(u) d u, \\
\int_{-\infty}^{+\infty} \phi(a)^{\frac{\alpha}{2}} d u<+\infty
\end{gathered}
$$

and $V(t, x)$ may depend on $t$.

It is clear that the preceding method may be applied, with:

$$
\begin{aligned}
& R_{a}(t, \tau)=R(t, \tau) \text { if }|\tau-t| \leqq a,=0 \text { if }|\tau-t|>a \\
& R_{a}^{\prime}(t, \tau)=R\left(t, \tau\left(-R_{a}(t, \tau)\right.\right.
\end{aligned}
$$

and so on. The limitations given by Lemma 3 and 4 are still valid; we start by considering, not $X(t)$, but its Laplacian limit in law $X^{*}(t)$; let $\gamma^{*}(u, v)=$ $E\left\{V\left[X^{*}(u)\right] V\left[X^{*}(v)\right]\right\}$ be the c of $Y^{*}(t)=V[t, X(t)]$, and

$$
L *(t, \tau)=\int_{t}^{\tau} Y *(u) d u
$$

It is easy to see that there is a function $K(\tau-t)$ such as:

$$
E\left(\left|L^{*}(t, \tau)\right|^{3}\right) \leqq K(\tau-t)
$$

is valid for every $(t, \tau)$. Consequently, by Lemma 1 , the analogue of theorem IV yields, if we suppose that:

$$
\liminf _{T \rightarrow+\infty} \frac{1}{T} \int_{t}^{t+T} \int_{t}^{t+T} \gamma^{*}(u, v) d u d v>0
$$

[this limit is independent of $t$; this assumption is to replace (19)]. And we may point out that we have for $X^{*}(t)$ a decomposition analogous to (24). But it is an open problem to characterize, directly on their c., the Laplacian processes such that there is a corresponding function $R(t, \tau)$ satisfying the preceding assumptions. But we may mention that, in the electrical applications it is known a priori that the interfering Laplacian processes are of the above considered kind.

Now, if we take the case of $X(t)$ instead of $X^{*}(t)$, we may follow exactly the same procedure; the only exception is that, now, the limitation analogous to (25) is not automatically satisfied, and we need a supplementary assumption, like, for instance: 


$$
E\left[\left(\int_{-\infty}^{+\infty} R(t-\tau)\left|d N^{*}(\tau)\right|\right)^{3}\right]<+\infty
$$

in order to have (25).

5 . On the other hand, it would be useful to have an assumption on $V$ weaker than (11); considering only the stationary case, it is easy to see that (11) may be replaced by the weaker assumption that:

$$
\left|V(x)-V\left(x^{\prime}\right)\right| \leqq \lambda(x)\left|x-x^{\prime}\right|^{\alpha} \quad(0 \leqq \alpha<1)
$$

where the positive function of $x \lambda(x)$ is such as:

$$
E\left(|\lambda[X(t)]|^{\frac{2}{1-\alpha}}\right)<+\infty
$$

or

$$
E\left(\left|\lambda\left[X^{*}(t)\right]\right|^{\frac{2}{1-\alpha}}\right)<+\infty
$$

If $\lambda(x)$ is bounded by:

$$
\lambda(x) \leqq A+B|x|^{\beta}
$$

when $A, B, \beta$ are any positive numbers, (28) is always satisfied.

If $\alpha=1$, reasonings have to be slightly modified, but it is readily seen that weaker assumptions like the preceding one may be accepted.

But it would be useful to have assumptions such that $V$ may have some discontinuities.

Los Angeles, December 18, 1951. 